\title{
Annual Change in Pulmonary Function and Clinical Characteristics of Combined Pulmonary Fibrosis and Emphysema and Idiopathic Pulmonary Fibrosis: Over a 3-Year Follow-up
}

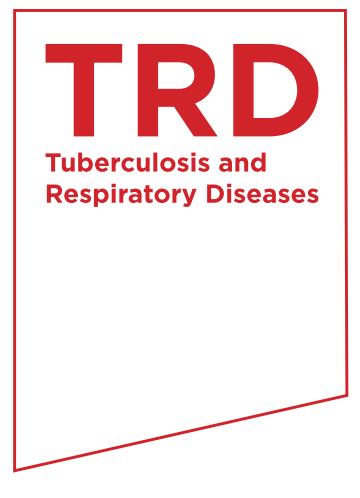

Yu Jin Kim, M.D. ${ }^{1}$, Seong Hyun Shin ${ }^{2}$, Jeong-Woong Park, M.D. ${ }^{1}$, Sun Young Kyung, M.D. ${ }^{1}$, Shin Myung Kang, M.D. ${ }^{1}$, Sang-Pyo Lee, M.D., , Yon Mi Sung, M.D. ${ }^{3}$, Yoon Kyung Kim, M.D. ${ }^{3}$ and Sung Hwan Jeong, M.D. ${ }^{1}$

${ }^{1}$ Division of Pulmonology, Department of Internal Medicine, ${ }^{2}$ Gachon University of Medicine and Science, ${ }^{3}$ Department of Radiology, Gachon University Gil Medical Center, Incheon, Korea

Background: Combined pulmonary fibrosis and emphysema (CPFE) have different pulmonary function tests (PFTs) and outcomes than idiopathic pulmonary fibrosis (IPF). The intention of this study was to identify unknown differences between CPFE and IPF by a retrospective comparison of clinical data including baseline and annual changes in pulmonary function, comorbidities, laboratory findings, clinical characteristics and cause of hospitalization.

Methods: This study retrospectively enrolled patients with CPFE and IPF who had undergone PFTs once or several times per year during a follow-up period of three years. Baseline clinical characteristics and the annual changes in the pulmonary function during the follow-up period were compared between 26 with CPFE and 42 patients with IPF.

Results: The baseline ratio of forced expiratory volume in one second to forced vital capacity $\left(\mathrm{FEV}_{1} / \mathrm{FVC} \%\right)$ in patients with CPFE was lower than that in patients with IPF ( $78.6 \pm 1.7$ vs. $82.9 \pm 1.1, \mathrm{p}=0.041)$. The annual decrease in $\mathrm{FEV}_{1} / \mathrm{FVC}$ in the CPFE was significantly higher than in the IPF. The annual decreases in diffusion capacity of carbon monoxide and FVC showed no significant differences between the two groups. The symptom durations of cough and sputum were in the CPFE significantly lower than in the IPF. The serum erythrocyte sedimentation rate level at the acute stage was significantly higher than in the IPF. There were no significant differences in the hospitalization rate and pneumonia was the most common cause of hospitalization in both study groups.

Conclusion: The annual decrease of $\mathrm{FEV}_{1} / \mathrm{FVC}$ was in patients with $\mathrm{CPFE}$ significantly higher than in the patients with IPF.

Keywords: Pulmonary Emphysema; Pulmonary Fibrosis; Idiopathic Pulmonary Fibrosis; Respiratory Function Tests

Address for correspondence: Sung Hwan Jeong, M.D.

Division of Pulmonology, Department of Internal Medicine, Gachon University Gil Hospital, 21 Namdong-daero 774beon-gil, Namdong-gu, Incheon 405-760, Korea

Phone: 82-32-460-2634, Fax: 82-32-469-4320, E-mail: jsw@gilhospital.com

Received: Mar. 24, 2014

Revised: May 7, 2014

Accepted: May 28, 2014

@a) It is identical to the Creative Commons Attribution Non-Commercial License (http://creativecommons.org/licenses/by-nc/3.0/).

Copyright $\odot 2014$ The Korean Academy of Tuberculosis and Respiratory Diseases. All rights reserved. 


\section{Introduction}

Combined pulmonary fibrosis and emphysema (CPFE) is defined as concurrent emphysema and pulmonary fibrosis in a patient. This disease may have a different clinical outcome and pulmonary function compared to idiopathic pulmonary fibrosis (IPF). But the difference and magnitude have not been completely elucidated. CPFE experience mildly expiratory flow limitation and overexpansion, as well as significantly compromised gas exchange during exercise ${ }^{1-9}$. Notably, CPFE have a relatively normal lung volume compared to those with $\mathrm{IPF}^{2-4,10}$. Pulmonary emphysema increases lung compliance and volume, while pulmonary fibrosis decreases compliance and volume. Thus, ventilatory function appears relatively normal because of these two opposing conditions in $\mathrm{CPFE}^{4,11}$. Emphysema associated with CPFE is localized to the upper pulmonary lobes and pulmonary fibrosis to the lower lobes ${ }^{4}$. CPFE is often concomitant with numerous complications including pulmonary hypertension, acute lung injury, and lung cance ${ }^{4,5,12-14}$. The condition occurs primarily in male smokers, and these co-morbidities are known to impact the survival rate $^{4-6,12}$. Generally, pulmonary function is comparatively normal in CPFE than in IPF. In a prior study evaluating the long term pulmonary function in CPFE and IPF, the reduction in vital capacity and total lung capacity was less in CPFE patients than in IPF; the diffusion capacity of carbon monoxide (DLco) was also lower in the CPFE than the IPF ${ }^{11}$. At present, there are few long-term studies comparing the change in pulmonary function between CPFE and $\mathrm{IPF}^{3,6,11,15}$. Comparative studies focused on other clinical parameters such as acute exacerbation are similarly inadequate. We hypothesized that CPFE present distinct clinical characteristics and disease progression than IPF in pulmonary function and acute exacerbation. We investigated the correlation to several clinical parameters including concomitant diseases, pulmonary function, laboratory findings, symptoms, and hospitalization to discern potential differences between the two diseases that have not been revealed.

This protocol was screened and approved by the Committee of Clinical Research Ethics of Gachon University's Gil Medical Center.

\section{Materials and Methods}

\section{Patient characteristics}

CPFE and IPF patients receiving pulmonary function test (PFT) over 3 years at the Division of Pulmonology at Gachon University Gil Medical Center from 1 January 2000 to 31 June 2013 were retrospectively reviewed. In total, 68 records of 26 patients with CPFE and 42 patients with IPF were analyzed for sex, age, smoking status, laboratory findings, and pulmonary function. IPF was diagnosed based on the American Thoracic
Society (ATS) and European Respiratory Society (ERS) 2012 guidelines ${ }^{16}$. CPFE was diagnosed in patients showing welldemarcated areas of low attenuation typical of emphysema located predominantly in the upper lung zones and patterns compatible with IPF ${ }^{11}$.

\section{Clinical characteristics and PFT}

The sex, age, smoking status, and laboratory findings at the first medical examination were screened, and the clinical symptoms, symptom duration, hospitalization incidence and cause, erythrocyte sedimentation rate (ESR) and C-reactive protein (CRP) during stable periods, and the ESR and CRP during hospitalization periods during the follow-up period were compared between the patient groups. The follow-up period was defined as the interval between the first hospital visit and the last visit or patient death. The PFT evaluated the forced expiratory volume in one second $\left(\mathrm{FEV}_{1}\right)$, forced vital capacity (FVC), and pulmonary DLco. Data were measured as the percentage (\% predicted) of the absolute $(\mathrm{mL})$ and estimated values. Pulmonary function at the initial hospital visit served as the baseline respiratory function. Patients who performed a PFT at least three times during the study period.

\section{Statistical analysis}

Data means and magnitude of change in numerical pulmonary function and laboratory findings were compared using the t-test. Correlations between the binary variables such as sex, age, smoking, and concomitant diseases were analyzed using the chi-square test. Patients who performed a PFT at least three times during the study period were reviewed to evaluate the pulmonary function change over time using linear regression analysis. Differences were considered significant when the p-value less than 0.05 .

\section{Results}

\section{Clinical characteristics of CPFE and IPF (Table 1)}

Male accounted for 23 (88.5\%) of the CPFE and was significantly higher than that the IPF ( $\mathrm{p}=0.007)$. Smoking status and pack-year were significantly higher in the CPFE $(\mathrm{p}=0.001)$. In the analysis of respiratory symptoms (excertional dyspnea, cough, and sputum), there were no significant differences between the two groups. The mean symptom duration for both cough and sputum were significantly longer in the IPF than in the CPFE ( $p=0.007$ and $p=0.018$ ). There were no significant differences between the two groups in $\mathrm{FVC}$ and $\mathrm{FEV}_{1}$, but the $\mathrm{FEV}_{1} / \mathrm{FVC}$ ratio was significantly lower in the CPFE patient group ( $\mathrm{p}=0.041$ ). DLco (\%) were lower in the CPFE compared to the IPF, but the difference was not statistically significant. 
Table 1. Clinical characteristics of CPFE and IPF

\begin{tabular}{|lccc|}
\hline & CPFE (n=26) & IPF (n=42) & p-value \\
\hline Sex (male/female) & $23 / 3$ & $24 / 18$ & 0.007 \\
Age, yr & $67.6 \pm 2.2$ & $68.2 \pm 1.7$ & 0.822 \\
Follow up, mo & $48.9 \pm 6.8$ & $62.1 \pm 6.7$ & 0.176 \\
Survival (no) & 2 & 9 & 0.780 \\
Smoking (yes/no) & $24 / 2$ & $19 / 23$ & 0.001 \\
BMI, kg/m ${ }^{2}$ & $23.4 \pm 0.5$ & $23.9 \pm 0.5$ & 0.488 \\
Pack-year & $28.3 \pm 4.1$ & $10.7 \pm 2.5$ & 0.001 \\
DOE (yes) & 19 & 32 & 0.485 \\
Cough (yes) & 23 & 38 & 0.452 \\
Sputum (yes) & 17 & 26 & 0.514 \\
DOE, wk & $54.5 \pm 9.6$ & $63.2 \pm 7.1$ & 0.473 \\
Cough, wk & $39.9 \pm 6.6$ & $65.8 \pm 6.5$ & 0.007 \\
Sputum, wk & $35.5 \pm 6.7$ & $63 \pm 8.0$ & 0.018 \\
Baseline FVC, L & $2.9 \pm 0.2$ & $2.6 \pm 0.2$ & 0.194 \\
Baseline FVC, \% & $79.3 \pm 4.0$ & $79.7 \pm 3.0$ & 0.934 \\
Baseline FEV ${ }_{1}, \mathrm{~L}$ & $2.3 \pm 0.2$ & $2.1 \pm 0.1$ & 0.404 \\
Baseline FEV,$\%$ & $85.7 \pm 5.6$ & $93.6 \pm 3.5$ & 0.238 \\
Baseline DLco, \% & $76.5 \pm 6.1$ & $82.8 \pm 4.3$ & 0.398 \\
Baseline FEV ${ }_{1} / \mathrm{FVC}, \%$ & $78.6 \pm 1.7$ & $82.9 \pm 1.1$ & 0.041 \\
Systolic PAP, mm Hg & $32.7 \pm 4.0$ & $39.3 \pm 3.9$ & 0.711 \\
\hline
\end{tabular}

Values are presented as number or mean \pm SD.

CPFE: combined pulmonary fibrosis and emphysema; IPF: idiopathic pulmonary fibrosis; BMI: body mass index; DOE: dyspnea of exertion; FVC: forced vital capacity; $\mathrm{FEV}_{1}$ : forced expiratory volume in 1 second; DLco: diffusion capacity of carbon monoxide; PAP: pulmonary arterial pressure; SD: standard deviation.

Both groups exhibited an elevated pulmonary artery pressure compared with normal range, but there was no significant difference between the two groups. We observed no significant difference in survival both group (Figure 1).

\section{Annual changes in pulmonary function in the CPFE and IPF (Table 2)}

Notably, the $\mathrm{FEV}_{1} / \mathrm{FVC}$ did significantly decrease over time in the CPFE compared with the IPF ( $\mathrm{p}=0.007$ ). In the CPFE, the mean FVC increased over time, while the mean $\mathrm{FEV}_{1}$ decreased. In the IPF, FVC decreased, and the $\mathrm{FEV}_{1}$ slightly increased over time. However, there were no significant differences in $\mathrm{FVC}$ or $\mathrm{FEV}_{1}$ change over time between the two groups. DLco decreased in both groups over, but to a greater extent in CPFE; however, there was no significant difference between the groups.

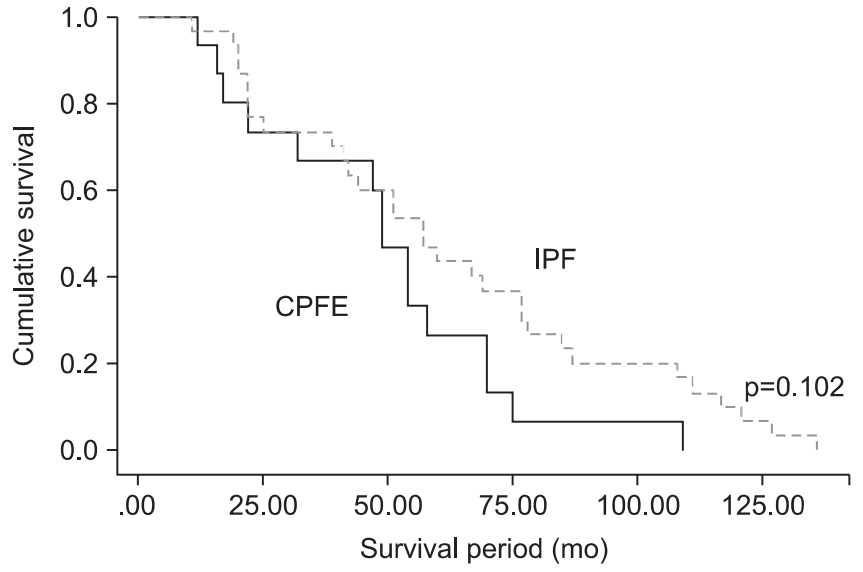

Figure 1. Kaplan-Meier survival curves. CPFE: combined pulmonary fibrosis and emphysema; IPF: idiopathic pulmonary fibrosis.

Table 2. Annual changes in pulmonary function in the groups with CPFE and IPF

\begin{tabular}{|lccc|}
\hline \multicolumn{1}{|c}{ CPFE } & IPF & p-value \\
\hline $\mathrm{FVC}, \mathrm{mL} / \mathrm{yr}$ & $47 \pm 44$ & $56 \pm 50$ & 0.150 \\
$\mathrm{FVC}, \% / \mathrm{yr}$ & $-0.833 \pm 1.693$ & $0.667 \pm 1.160$ & 0.475 \\
$\mathrm{FEV}_{1}, \mathrm{~mL} / \mathrm{yr}$ & $82 \pm 42$ & $21 \pm 29$ & 0.058 \\
$\mathrm{FEV}_{1}, \% / \mathrm{yr}$ & $0.323 \pm 3.399$ & $1.577 \pm 1.094$ & 0.732 \\
$\mathrm{DL}_{\mathrm{CO}}, \mathrm{mL} / \mathrm{min} / \mathrm{mm} \mathrm{Hg} / \mathrm{yr}$ & $-1.062 \pm 0.855$ & $-0.362 \pm 0.600$ & 0.512 \\
$\mathrm{DL}_{\mathrm{CO}}, \% / \mathrm{yr}$ & $-0.705 \pm 2.946$ & $-4.775 \pm 3.931$ & 0.417 \\
$\mathrm{FEV}_{1} / \mathrm{FVC}, 1 / \mathrm{yr}$ & $-0.036 \pm 0.013$ & $0.031 \pm 0.018$ & 0.007 \\
\hline
\end{tabular}

Values are presented as mean \pm SD or number.

CPFE: combined pulmonary fibrosis and emphysema; IPF: idiopathic pulmonary fibrosis; FVC: forced vital capacity; $\mathrm{FEV}_{1}$ : forced expiratory volume in 1 second; $\mathrm{DL}_{\mathrm{CO}}$ : diffusion capacity of carbon monoxide; SD: standard deviation.

Table 3. Comorbidity of CPFE and IPF

\begin{tabular}{|lccc|}
\hline & CPFE (n=26) & IPF (n=42) & p-value \\
\hline TB (yes/no) & $4 / 22$ & $9 / 33$ & 0.752 \\
DM (yes/no) & $12 / 14$ & $14 / 28$ & 0.316 \\
Hypertension (yes/no) & $9 / 17$ & $20 / 22$ & 0.324 \\
Cardiac disease (yes/no) & $8 / 18$ & $10 / 32$ & 0.579 \\
Cerebral disease (yes/no) & $3 / 23$ & $2 / 40$ & 0.363 \\
Lung cancer (yes/no) & $2 / 24$ & $3 / 39$ & 0.933 \\
\hline
\end{tabular}

CPFE: combined pulmonary fibrosis and emphysema; IPF: idiopathic pulmonary fibrosis; TB: Mycobacterium tuberculosis history; DM: diabetes mellitus.

\section{Co-morbidities of CPFE and IPF (Table 3)}

In the analysis of co-morbidities such as tuberculosis, dia- 
betes, hypertension, heart disease, cerebral disease, and lung cancer to survival rate, there were no significant differences in any of the variables between the two groups.

\section{Laboratory finding of CPFE and IPF (Table 4)}

The mean ESR during hospital period was significantly higher in the IPF ( $p=0.029)$. The mean ESR during stable periods and the mean CRP during hospital period and stable period were not significantly different between the two

Table 4. Laboratory findings of CPFE and IPF

\begin{tabular}{|c|c|c|c|}
\hline & CPFE $(n=26)$ & IPF $(n=26)$ & p-value \\
\hline $\mathrm{PaO}_{2}, \mathrm{~mm} \mathrm{Hg}$ & $84.1 \pm 5.3$ & $91.6 \pm 4.2$ & 0.277 \\
\hline $\mathrm{PaCO}_{2}, \mathrm{~mm} \mathrm{Hg}$ & $36.4 \pm 4.1$ & $32.5 \pm 1.0$ & 0.357 \\
\hline \multicolumn{4}{|l|}{ ESR, $\mathrm{mm} / \mathrm{hr}$} \\
\hline Stable & $29.7 \pm 7.5$ & $25.5 \pm 2.8$ & 0.542 \\
\hline Exacerbation & $28.3 \pm 7.2$ & $51.8 \pm 6.9$ & 0.029 \\
\hline \multicolumn{4}{|l|}{ CRP, mg/dL } \\
\hline Stable & $0.1 \pm 0.1$ & $0.5 \pm 0.4$ & 0.375 \\
\hline Exacerbation & $6.2 \pm 1.5$ & $6.5 \pm 1.5$ & 0.87 \\
\hline LDH, U/L & $780.2 \pm 203.6$ & $551.4 \pm 46.4$ & 0.231 \\
\hline $\mathrm{CPK}, \mathrm{U} / \mathrm{L}$ & $286.8 \pm 125.9$ & $120.2 \pm 34.9$ & 0.165 \\
\hline Glucose, mg/dL & $124.4 \pm 10.9$ & $147.1 \pm 17.6$ & 0.281 \\
\hline Protein, g/dL & $7.3 \pm 0.1$ & $7.3 \pm 0.1$ & 0.961 \\
\hline Albumin, g/dL & $4.0 \pm 0.1$ & $3.9 \pm 0.1$ & 0.578 \\
\hline T-bilirubin, mg/dL & $0.6 \pm 0.0$ & $0.6 \pm 0.0$ & 0.636 \\
\hline AST, U/L & $44.1 \pm 12.5$ & $31.3 \pm 3.2$ & 0.238 \\
\hline ALT, U/L & $34.5 \pm 10.3$ & $27.9 \pm 3.6$ & 0.551 \\
\hline ALP, U/L & $85.2 \pm 8.7$ & $115.3 \pm 12.4$ & 0.088 \\
\hline Ca, mg/dL & $8.6 \pm 0.1$ & $9.0 \pm 0.1$ & 0.025 \\
\hline $\mathrm{Mg}, \mathrm{mg} / \mathrm{dL}$ & $2.2 \pm 0.2$ & $2.3 \pm 0.1$ & 0.817 \\
\hline $\mathrm{Hb}, \mathrm{g} / \mathrm{dL}$ & $13.4 \pm 0.4$ & $13.2 \pm 0.3$ & 0.627 \\
\hline $\mathrm{WBC}, 10^{-3} / \mathrm{mm}^{-3}$ & $9.2 \pm 0.9$ & $9.1 \pm 0.6$ & 0.996 \\
\hline Lymphocyte, \% & $28.4 \pm 2.0$ & $27.1 \pm 1.7$ & 0.626 \\
\hline PLT, $10^{-3} / \mathrm{mm}^{-3}$ & $246.6 \pm 13.0$ & $280.9 \pm 19.3$ & 0.145 \\
\hline $\mathrm{HbA}_{1 \mathrm{C}}, \%$ & $6.8 \pm 0.3$ & $8.1 \pm 1.0$ & 0.190 \\
\hline ProBNP, pg/mL & $243.8 \pm 99.3$ & $794.8 \pm 435.9$ & 0.241 \\
\hline
\end{tabular}

Values are presented as mean \pm SD or number.

CPFE: combined pulmonary fibrosis and emphysema; IPF: idiopathic pulmonary fibrosis; ESR: erythrocyte sedimentation rate; CRP: C-reactive protein; $\mathrm{LDH}$ : lactate dehydrogenase; CPK: creatine kinase; T-bilirubin: total bilirubin; AST: aspartate aminotransferase; ALT: alanine transaminase; ALP: alkaline phosphatase; WBC: white blood cell; PLT: platelet; HbAlC: hemoglobinA1C; ProBNP: pro-brain natriuretic peptide; SD: standard deviation. groups. The mean baseline serum calcium concentration at stable was higher in the IPF than the CPFE but was within the normal range $(\mathrm{p}=0.025)$. There was no significant difference between the two groups in the other laboratory findings.

\section{Cause of hospitalization (Table 5)}

The rate of hospitalization was $38 \%$ in the CPFE and $45 \%$ in the IPF, indicating no significant difference between the two groups. Pneumonia, which occurred at the highest frequency of hospitalization, accounted for $56 \%$ of CPFE and $56 \%$ of IPF, which was a high incidence in both groups.

\section{Treatment of CPFE and IPF (Table 6)}

Prednisolone was significantly difference compared with the CPFE and IPF $(\mathrm{p}=0.029)$. At a $64 \%$ prescription rate in IPF, which was significantly higher than that of CPFE at $42 \%$. There were no significant differences between the two groups in the prescription rate of other medications such as short acting $\beta$-agonists, short acting anticholinergics, theophylline, and $\mathrm{N}$ acetylcysteine.

Table 5. Causes of hospitalization

\begin{tabular}{|cccc|}
\hline & CPFE (n=26) & IPF (n=42) & p-value \\
\hline Cause of hospitalization & $10 / 26(38)$ & $20 / 42(45)$ & 0.314 \\
Pneumonia & $5(56)$ & $12(57)$ & 0.565 \\
Pneumothorax & $1(11)$ & $1(5)$ & 0.995 \\
Lung cancer & $1(11)$ & $1(5)$ & 0.995 \\
Others* & $2(22)$ & $7(33)$ & 0.465 \\
\hline
\end{tabular}

Values are presented as number (\%).

*Upper airway infection, pulmonary thromboembolism, Still's disease, acute exacerbation of IPF.

CPFE: combined pulmonary fibrosis and emphysema; IPF: idiopathic pulmonary fibrosis.

Table 6. Treatments of CPFE and IPF

\begin{tabular}{|lrrr|}
\hline \multicolumn{1}{|c}{ CPFE (n=26) } & IPF (n=42) & p-value \\
\hline Inhaled bronchodilator & & & \\
\hline Short acting $\beta$-agonist & $3(11.5)$ & $7(16.7)$ & 0.562 \\
Short acting anticholinergic & $4(15.4)$ & $12(28.6)$ & 0.213 \\
Prednisolone & $11(42.3)$ & $29(64.0)$ & 0.029 \\
N-acetylcysteine & $22(84.6)$ & $37(88.1)$ & 0.681 \\
Theophylline & $6(23.1)$ & $5(11.9)$ & 0.224 \\
\hline
\end{tabular}

Values are presented as number (\%).

CPFE: combined pulmonary fibrosis and emphysema; IPF: idiopathic pulmonary fibrosis. 


\section{Discussion}

Compared to the baseline PFT, the $\mathrm{FEV}_{1} / \mathrm{FVC}$ ratio was significantly different between the two groups and was lower in CPFE than in IPF, which is consistent with previous reports ${ }^{2,11,15}$. Cottin et al. ${ }^{2}$ reported that the $\mathrm{FEV}_{1} / \mathrm{FVC}$ in CPFE was lower than the normal range. Mura et al. ${ }^{3}$ asserted that the existence and range level are important factors promoting decline in pulmonary function including $\mathrm{FEV}_{1} / \mathrm{FVC}$. Akagi et al. ${ }^{11}$ also observed that the baseline $\mathrm{FEV}_{1} / \mathrm{FVC}$ in CPFE was decreased compared to $\mathrm{FEV}_{1} / \mathrm{FVC}$ in IPF. Also, they found that the baseline DLco and DLco was decreased to a significantly higher in the CPFE than in the $\operatorname{IPF}^{11}$. But this study report did not reveal a significant difference in the baseline DLco of either group. Moreover, there was no statistically significant difference in the FVC decrease. Over the study followup period, change in the $\mathrm{FEV}_{1} / \mathrm{FVC}$ was disparate between the two groups, a finding similar to that reported by previous study ${ }^{11,15}$. The $\mathrm{FEV}_{1} / \mathrm{FVC}$ in IPF remained mostly consistent over the follow-up period, but the ratio significantly decreased in the CPFE during the follow-up period ${ }^{11}$. Distinct from IPF, CPFE was associated with a progressively obstructive pattern over time, which highlights the importance of bronchodilator therapy in CPFE. In a study comparing CPFE and COPD executed by Kitaguchi et al. ${ }^{15}$, the $\mathrm{FEV}_{1} / \mathrm{FVC}$ in CPFE tended to decreased during the follow-up period, like the our study. Patients with CPFE are commonly men with smoking history. It seems most likely that emphysema affected $\mathrm{FEV}_{1} / \mathrm{FVC}$ decline by the effect of the fibrosis. While Akagi et al. ${ }^{11}$ reported a significant yearly decline in DLco between the two groups, our data did not show such a trend, indicating the need for additional research. Notably, this study revealed a significantly long duration of cough and sputum in the IPF compared to the CPFE. Moreover, the mean ESR during hospitalization was higher in the IPF. Previous studies reported that CPFE and IPF have similar mortality ${ }^{8,17}$. But Kurashima et al. ${ }^{6}$ reported CPFE had better survival compared with IPF. ESR and CRP might show as systemic inflammatory marker in chronic obstructive pulmonary disease ${ }^{18}$. CRP increases as $\mathrm{FEV}_{1} \%$ worsens ${ }^{19}$. It is still uncertain the role of inflammation in IPF. But Mura et al. ${ }^{20}$ suggested inflammatory activity in IPF is relavant. CPFE have different clinical features such as relation with emphysema and smoking history compared with IPF. So the mean ESR during hospitalization was higher in the IPF. ESR might show as a marker of systemic inflammation in CPFE and IPF. Further study needed whether ESR and the symptom duration may be prognostic indicator. Both groups had a pulmonary artery pressure higher than the normal range, but there was no significant difference between the groups. This result is similar to previous reports showing that the pulmonary hypertension incidence is higher in CPFE patients ${ }^{5,17}$. Additional prospective studies are needed to clarify the relationship between pulmonary hypertension and CPFE. There are several limitations in this study. First, this is single-center and retrospective study. Second, the assessment of emphysema did not use scoring method of emphysema. Third, we did not measure the exact areas of fibrosis. But, this study showed that the presence of concurrent emphysema in IPF is important factor about prognosis and PFT changes of patient. Further studies investigating the relationship between the obstructive change and short symptom duration in CPFE and prognosis are needed. The study of inflammatory mediators and biomarker in CPFE also merits evaluation through prospective studies.

In conclusion, Annual decrease in $\mathrm{FEV}_{1} / \mathrm{FVC}$ in CPFE was significantly more than IPF. In addition, this is the report showing the yearly dynamics changes of pulmonary function parameters, and analysis of the frequency and causes of hospitalization in CPFE compared with IPF.

\section{Conflicts of Interest}

No potential conflict of interest relevant to this article was reported.

\section{References}

1. American Thoracic Society; European Respiratory Society. American Thoracic Society/European Respiratory Society International Multidisciplinary Consensus Classification of the Idiopathic Interstitial Pneumonias. This joint statement of the American Thoracic Society (ATS), and the European Respiratory Society (ERS) was adopted by the ATS board of directors, June 2001 and by the ERS Executive Committee, June 2001. Am J Respir Crit Care Med 2002;165:277-304.

2. Cottin V, Nunes H, Brillet PY, Delaval P, Devouassoux G, Tillie-Leblond I, et al. Combined pulmonary fibrosis and emphysema: a distinct underrecognised entity. Eur Respir J 2005;26:586-93.

3. Mura M, Zompatori M, Pacilli AM, Fasano L, Schiavina M, Fabbri M. The presence of emphysema further impairs physiologic function in patients with idiopathic pulmonary fibrosis. Respir Care 2006;51:257-65.

4. Jankowich MD, Rounds SI. Combined pulmonary fibrosis and emphysema syndrome: a review. Chest 2012;141:222-31.

5. Grubstein A, Bendayan D, Schactman I, Cohen M, Shitrit D, Kramer MR. Concomitant upper-lobe bullous emphysema, lower-lobe interstitial fibrosis and pulmonary hypertension in heavy smokers: report of eight cases and review of the literature. Respir Med 2005;99:948-54.

6. Kurashima K, Takayanagi N, Tsuchiya N, Kanauchi T, Ueda M, Hoshi T, et al. The effect of emphysema on lung function and survival in patients with idiopathic pulmonary fibrosis. Respirology 2010;15:843-8.

7. Collard HR, King TE Jr, Bartelson BB, Vourlekis JS, Schwarz 
MI, Brown KK. Changes in clinical and physiologic variables predict survival in idiopathic pulmonary fibrosis. Am J Respir Crit Care Med 2003;168:538-42.

8. Jankowich MD, Rounds S. Combined pulmonary fibrosis and emphysema alters physiology but has similar mortality to pulmonary fibrosis without emphysema. Lung 2010;188:36573.

9. Kitaguchi Y, Fujimoto K, Hanaoka M, Kawakami S, Honda T, Kubo K. Clinical characteristics of combined pulmonary fibrosis and emphysema. Respirology 2010;15:265-71.

10. Schmidt SL, Nambiar AM, Tayob N, Sundaram B, Han MK, Gross BH, et al. Pulmonary function measures predict mortality differently in IPF versus combined pulmonary fibrosis and emphysema. Eur Respir J 201 1;38:176-83.

11. Akagi T, Matsumoto T, Harada T, Tanaka M, Kuraki T, Fujita M, et al. Coexistent emphysema delays the decrease of vital capacity in idiopathic pulmonary fibrosis. Respir Med 2009;103:1209-15.

12. Mejia M, Carrillo G, Rojas-Serrano J, Estrada A, Suarez T, Alonso D, et al. Idiopathic pulmonary fibrosis and emphysema: decreased survival associated with severe pulmonary arterial hypertension. Chest 2009;136:10-5.

13. Usui K, Tanai C, Tanaka Y, Noda H, Ishihara T. The prevalence of pulmonary fibrosis combined with emphysema in patients with lung cancer. Respirology 2011;16:326-31.

14. Lim TK. Respiratory failure from combined emphysema and pulmonary fibrosis. Singapore Med J 1993;34:169-71.
15. Kitaguchi Y, Fujimoto K, Hayashi R, Hanaoka M, Honda T, Kubo K. Annual changes in pulmonary function in combined pulmonary fibrosis and emphysema: over a 5-year follow-up. Respir Med 2013;107:1986-92.

16. Raghu G, Collard HR, Egan JJ, Martinez FJ, Behr J, Brown KK, et al. An official ATS/ERS/JRS/ALAT statement: idiopathic pulmonary fibrosis: evidence-based guidelines for diagnosis and management. Am J Respir Crit Care Med 2011;183:788824.

17. Ryerson CJ, Hartman T, Elicker BM, Ley B, Lee JS, Abbritti M, et al. Clinical features and outcomes in combined pulmonary fibrosis and emphysema in idiopathic pulmonary fibrosis. Chest 2013;144:234-40.

18. Corsonello A, Pedone C, Battaglia S, Paglino G, Bellia V, Incalzi RA. C-reactive protein (CRP) and erythrocyte sedimentation rate (ESR) as inflammation markers in elderly patients with stable chronic obstructive pulmonary disease (COPD). Arch Gerontol Geriatr 2011;53:190-5.

19. Tkacova R, Kluchova Z, Joppa P, Petrasova D, Molcanyiova A. Systemic inflammation and systemic oxidative stress in patients with acute exacerbations of COPD. Respir Med 2007;101:1670-6.

20. Mura M, Belmonte G, Fanti S, Contini P, Pacilli AM, Fasano $\mathrm{L}$, et al. Inflammatory activity is still present in the advanced stages of idiopathic pulmonary fibrosis. Respirology 2005;10:609-14. 